\section{Tätigkeitsbericht der Glaukom-Kommission der ÖOG (Mai 2003-Mai 2004)}

Mitglieder: Univ.-Doz. Andrea Mistlberger - Vorsitzende OA Dr. Anton Hommer - Schriftuürer

Dr. Martin Eckhardt

Univ.-Prof. Dr. Christoph Faschinger

Dr. Petra Freigassner

OA Dr. Wolfgang Kuess

Univ.-Prof. Dr. Georg Rainer

OA Dr. Karl Rigal

OA Dr. Barbara Teuchner

Univ.-Prof. Dr. Clemens Vass

Zusammenstellung der Aktivitäten:

\section{Sitzungstermine: September 2003}

\section{Jänner, Februar 2004}

Mai 2004

\section{Glaukomhotline - Radiosprechstunde:}

Jeden ersten Montag im Monat steht ein Mitglied der Kommission für eine Stunde am Telefon Rede und Antwort $z \mathfrak{u}$ Glaukomfragen! Österreichweit gute Akzeptanz. Interessanterweise sind neben allgemeinen Fragen immer mehr Detailfragen zu beantworten, was doch auf eine zunehmende Information der Bevölkerung schließen lässt. Tel. 01/4097502

\section{Bericht der Kommission für Internationale Ophthalmologie}

Am 18. 5. 2004 fand in Alpbach, Tirol die konstituierende Sitzung der Kommission für Internationale Ophthalmologie (KIO) statt. Die Gründung dieser Kommission geht auf eine Idee von MR Dr. Heinz Derka zurück und wurde von Prof. Dr. Günther Grabner, dem ständigen wissenschaftlichen Sekretär der ÖOG initiiert.

\section{Mitglieder der Kommission:}

Prof. Dr. Talin Barisani-Asenbauer

(stellvertretende Vorsitzende)

Prim. Prof. Dr. Susanne Binder

MR Dr. Heinz Derka

Dr. Johann Dillinger, Vorstandsmitglied von „Ostafrikakreis“, Traunstein, Bayern

Prof. Dr. Christoph Faschinger,

Vorstandsmitglied von „Sehen ohne Grenzen“

MR Dr. Roderich Fellner

Dr. Peter Gorka

Prim. Prof Dr. Günther Grabner

Dr. Alois Kolorz, Ärztekammervertreter bei der UEMS

Prof. Dr. Werner Kulnig

Prim. Prof. Dr. Siegfried Priglinger,

Vorstandsmitglied von ,Licht für die Welt/CBM“

Dr. Karl Rigal,

Vorstandsmitglied von ,Licht für die Welt/CBM“"

(Vorsitzender)

Mag. Rupert Roniger,

Geschäftsführer von ,Licht für die Welt/CBM“

Dr. Robert Schneider

Dr. Gerhard Schuhmann,

Vorstandsmitglied von „Licht für die Welt/CBM“
3. Vorträge und Fortbildungsveranstaltungen der Mitglieder:

Praxisrelevanter Glaukomworkshop (September 2003)

Wintersymposium Glaukom (Februar 2004): Themen wie Neuroprotektion, diverse operative Verfahren, Workshops $\mathrm{zu}$ verschiedenen Glaukomspielformen

Weitblick-Wochenende (April 2004): Glaukom im Wandel der Zeit, Ökonomie, Sparzwang versus therapeutische Notwendigkeit, sowie rechtliche Aspekte

Teilnahmen an diversen Advisory Board Meetings - Verbindung mit der Europäischen Glaukomgesellschaft

Vorträge bei Fachgruppensitzung

Vorträge in den Bundesländern bei praktischen Ärzten

\section{Neuauflage eines Glaukompasses:}

gesponsert durch die Fa. MSD, über diese sind auch bei Bedarf weitere Glaukompässe für die Verwendung in der Ordination zu beziehen

\section{Neues Konsensuspaper 2004}

„Diagnose und Therapie des Glaukoms 2004" wurde mit Unterstützung von Update Europe (Gesellschaft für ärztliche Fortbildung $\mathrm{GmbH}$ ) erstellt, großzügigerweise wurden sämtliche Kosten von der Fa. Pfizer übernommen.

Das Konsensuspaper wurde im Mai 2004 mit dem Spektrum der Augenheilkunde versandt.

DDr. Nikolaus Segur-Eltz

Johannes Trimmel,

Projektressortleiter von „Licht für die Welt/CBM“

(Schriftführer)

Als Aufgaben der KIO wurden festgelegt:

1) Vertretung der ÖOG beim European Board of Ophthalmology (EBO) und

Union of European Medical Scientists (UEMS)

2) Entwicklung einer Plattform für Entwicklungszusammenarbeit

\section{Erste Beschlüsse der KIO:}

ad 1)

- Entsendung von Prof. Dr. Talin Barisani-Asenbauer als Vertreterin der ÖOG zur UEMS

- Entsendung von Prof. Dr. Talin Barisani-Asenbauer und Prof. Dr. Werner Kulnig als Vertreter der ÖOG zum EBO $\operatorname{ad} 2$ )

- Entsendung von MR Dr. Heinz Derka als Vertreter der ÖOG zum 1. Österreichisch-Armenischen Ärztetreffen in Erewan, Armenien am 26. 6. 2004

- Kontaktaufnahme mit Prof. Dr. Daud Khan, dem Rektor des Khyber-Institutes of Ophthalmology und Vorstand des Pakistan Institute of Community Ophthalmology (PICO), der auch die erste offizielle Grußbotschaft an die KIO entrichtete

- Entwicklung der KIO zur Koordinationsstelle zwischen NGOs und Kollegen, die an Entwicklungszusammenarbeit interessiert sind

Als ersten Erfolg der Gründung der Kommission sehen wir das sehr bald bekundete Interesse von Kolleginnen und Kollegen, in der Kommission mitzuarbeiten. 